\title{
DLPNO-CCSD(T) Scaled Methods for the Accurate Treatment of Large Supramolecular Complexes
}

\author{
Joaquín Calbo, ${ }^{[a], *}$ Juan C. Sancho-García, ${ }^{[b]}$ Enrique Orti, ${ }^{[a]}$ Juan Aragó ${ }^{[a], *}$ \\ [a] Instituto de Ciencia Molecular, Universidad de Valencia, E-46980 Paterna, Spain \\ [b] Departamento de Química Física, Universidad de Alicante, E-03080 Alicante, Spain \\ Correspondence to: \\ Joaquín Calbo (E-mail: joaquin.calbo@uv.es) \\ Juan Aragó (E-mail: juan.arago@uv.es)
}

\section{ABSTRACT}

In this work, we present scaled variants of the DLPNO-CCSD(T) method, dubbed as (LS)DLPNO$\operatorname{CCSD}(T)$ and (NS)DLPNO-CCSD(T), to obtain accurate interaction energies in supramolecular complexes governed by noncovalent interactions. The novel scaled schemes are based on the linear combination of the DLPNO-CCSD(T) correlation energies calculated with the standard (LoosePNO and NormalPNO) and modified (Loose2PNO and Normal2PNO) DLPNO-CCSD(T) accuracy levels. The scaled DLPNO-CCSD(T) variants provide nearly TightPNO accuracy, which is essential for the quantification of weak noncovalent interactions, with a noticeable saving in computational cost. Importantly, the accuracy of the proposed schemes is preserved irrespective of the nature and strength of the supramolecular interaction. The (LS)DLPNO-CCSD(T) and (NS)DLPNO-CCSD(T) protocols have been used to study in depth the role of the $\mathrm{CH}-\pi$ versus $\pi-\pi$ interactions in the supramolecular complex formed by the electron-donor truxene-tetrathiafulvalene (truxTTF) and the electron-acceptor hemifullerene $\left(\mathrm{C}_{30} \mathrm{H}_{12}\right)$. (NS)DLPNO-CCSD(T)/CBS calculations clearly reveal the higher stability of staggered (dominated by $\mathrm{CH}-\pi$ interactions) versus bowl-in-bowl (dominated by $\pi-$ $\pi$ interactions) arrangements in the truxTTF $\bullet \mathrm{C}_{30} \mathrm{H}_{12}$ heterodimer. Hemifullerene and similar carbonbased buckybowls are therefore expected to self-assemble with donor compounds in a richer way other than the typical concave-convex $\pi-\pi$ arrangement found in fullerene-based aggregates.

\section{Introduction}

Noncovalent interactions ( $\mathrm{NCls}$ ), although being fairly weaker compared to covalent interactions, are one of the most important forces governing the association process in supramolecular complexes, and are extensively exploited for the self-assembly and molecular recognition of biomolecular systems. ${ }^{[1]}$ In the field of materials science, for instance, different electron-acceptor nanoforms of carbon, namely fullerenes, carbon nanotubes, and graphene, have been successfully combined by means of $\mathrm{NCls}$ with electron-donor systems to form donor-acceptor (D-A) supramolecular complexes. ${ }^{[2-9]}$ Experimental studies on these D-A supramolecular complexes are of great relevance to gain insight into the fundamental and omnipresent photoinduced electrontransfer process, which is required, for example, in organic photovoltaic applications. ${ }^{[10-13]}$

If we turn our attention to fullerenes, $\mathrm{C}_{60}$ and its derivatives, a large number of $\pi$-conjugated 
electron-donating systems are able to host the buckyball through $\pi-\pi$ intermolecular interactions, ${ }^{[5,7-8,14-16]}$ a particular kind of $\mathrm{NCls}$. Among the different donor moieties employed, the truxene-tetrathiafulvalene (truxTTF) system (Figure 1), based on a truxene core to which three dithiole rings are covalently attached, has been successfully used in the recognition of fullerene $C_{60}$ and $C_{70}$ due to their suitable concave-convex complementarity. ${ }^{[2,8]}$ Although $\mathrm{C}_{60}$ and $\mathrm{C}_{70}$ are by far the most used electronaccepting ( $n$-type) materials in, for instance, organic solar cells, the quest for novel and improved fullerene derivatives and/or fullerene fragments is a very active field of research. ${ }^{[17-19]}$ Recently, a combined theoretical and experimental study has revealed for the first time that the hemifullerene $\mathrm{C}_{30} \mathrm{H}_{12}$, a fragment of fullerene (Figure 1), is effectively hosted by truxTTF and mimics the electron-accepting behavior of fullerenes within the supramolecular truxTTF $\bullet \mathrm{C}_{30} \mathrm{H}_{12}$ complex. ${ }^{[10]}$ Fullerene fragments, in contrast to fullerenes, present terminal effects due to the presence of hydrogen atoms at the periphery. This subtle difference may give rise to $D-A$ supramolecular complexes not only dominated by $\pi-\pi$ intermolecular interactions, which is the main case for host-guest associations involving fullerenes, but also with participation of $\mathrm{CH}-\pi$ interactions. $^{[10]} \mathrm{CH}-\pi$ contacts are indeed crucial for understanding and explaining the structure of biological macromolecules, carbohydrates and molecular materials in the crystal. Infrared (IR) and nuclear magnetic resonance (NMR) spectroscopies provide experimental evidence of these elusive interactions. ${ }^{[20-22]} \quad \mathrm{D}-\mathrm{A}$ supramolecular structures with significant contribution of $\mathrm{CH}-\pi$ contacts have been proposed as the most stable structures for the truxTTF $\bullet \mathrm{C}_{30} \mathrm{H}_{12}$ associates. ${ }^{[10]}$ Experimental ${ }^{1} \mathrm{H}-\mathrm{NMR}$ evidences recently reported on similar truxTTF supramolecular complexes with corannulene-based buckybowls $\mathrm{C}_{32} \mathrm{H}_{12}$ and $\mathrm{C}_{38} \mathrm{H}_{14}$ point toward the formation of staggered associates dominated by $\mathrm{CH}-\pi$ interactions as the preferred arrangements. ${ }^{[11]}$ Nevertheless, interaction energy calculations using high-level $a b$ initio methods that support these supramolecular arrangements dominated by $\mathrm{CH}-\pi$ interactions have not been performed yet.

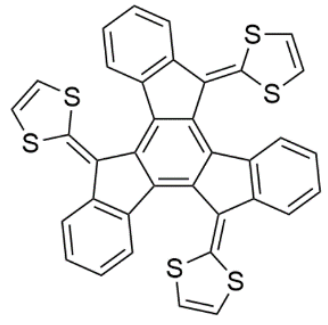

TruxTTF

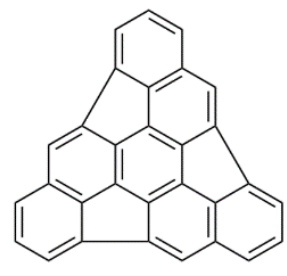

Hemifullerene $\mathrm{C}_{30} \mathrm{H}_{12}$
Figure 1. Chemical structure of the truxTTF and hemifullerene moieties.

The theoretical description of supramolecular D-A complexes involving fullerene fragments and buckybowls is still a difficult task, and requires the use of accurate and cost-effective quantum-chemical methods to account for $\mathrm{NCls}$ in a balanced way. Over the last years, the development of novel theoretical approaches to better understand the nature of the $\mathrm{NCls}$, and to compute accurate interaction energies for supramolecular assemblies of the most interest, has led to methodologies showing an unprecedented degree of accuracy in the context of noncovalent interactions. ${ }^{[23-25]} \mathrm{NCls}$ mainly arise from long-range electron correlation effects, a particular challenging problem that requires highly correlated wave function methods to provide an appropriate theoretical quantification of these elusive interactions. Coupled-cluster theory with single, double, and perturbatively connected triple excitations (CCSD(T)) has become the 'goldstandard' approximation used for the accurate treatment of these weak but important forces. ${ }^{[26]}$ However, the $\operatorname{CCSD}(T)$ method is computationally very demanding with a $\mathrm{O}\left(N^{7}\right)$ scaling, where $N$ is a measure of the system size, that prevents its use for medium to large "real-world" supramolecular complexes. On the other hand, the efficient domain-based local pair-natural orbital (DLPNO) approach developed by Neese et at. ${ }^{[27-28]}$ has recently 
been coupled to the $\operatorname{CSS}(T)$ method, offering a quantum-chemical method, dubbed as DLPNO$\operatorname{CCSD}(T)$, that exhibits the high accuracy of the semicanonical $\operatorname{CCSD}(T)$ but with a significantly reduced computational cost. ${ }^{[28]}$ The DLPNO$\operatorname{CCSD}(T)$ accuracy has been assessed on noncovalent complexes, ${ }^{[29]}$ enzymatic reactions, ${ }^{[30]}$ organic reactions, ${ }^{[30-31]}$ transitionmetal-promoted reactions, ${ }^{[32-34]}$ and even on a small protein. ${ }^{[28]}$ For instance, relative energies computed using the DLPNO-CCSD(T) approach provided an average error of approximately 0.5 $\mathrm{kcal} / \mathrm{mol}$ for a series of challenging test sets of medium-sized organic molecules. ${ }^{[35-36]}$ DLPNO$\operatorname{CCSD}(T)$ may therefore be viewed as a promising theoretical approximation able to provide accurate reference energies in large molecular systems for which other $a b$ initio methodologies become prohibitive.

Despite its success, the accuracy of the DLPNO-CCSD(T) approach, especially in the context of $\mathrm{NCls}$, significantly depends on its internal parameters or thresholds. The adjustable thresholds currently available in the most recent implementation ${ }^{[37]}$ are the PNO occupation number TCutPNO, the strong pair approximation cut-off TCutPairs, and the domain size parameter TCutMKN. Neese et al. tried to conceive the DLPNO-CCSD(T) as a blackbox tool and, therefore, this approach has been used at three different levels (termed as "LoosePNO", "NormaIPNO", and "TightPNO") according to the truncation thresholds (see Table 1 below for further details). ${ }^{[35]}$ Whereas the LoosePNO is demonstrated clearly sufficient for initial screening or exploration of a potential energy surface, NormalPNO is the accurate choice for most computational applications, such as general thermochemistry and thermochemical kinetics, and the TightPNO setup should be viewed as the level of choice for very accurate calculations, as needed in conformational equilibria or evaluation of $\mathrm{NCls.}{ }^{[33,35,38]}$ Interestingly, a recent study has shown that the accuracy of the NormalPNO setup can be improved with a reasonable (affordable) increase of computational cost by adjusting the cutoff threshold for the estimated pair correlation energies (TCutPairs) to $10^{-5}$ $E_{h}{ }^{[39]}$ However, an exploratory calibration of the PNO thresholds in accurate yet low-cost DLPNO-CCSD $(T)$ schemes for benchmarking and application-oriented purposes in supramolecular chemistry is still missing.

In this contribution, we propose two novel DLPNO-CCSD(T) variants, named as (LS)DLPNO$\operatorname{CCSD}(T)$ and (NS)DLPNO-CCSD(T), for the accurate treatment of large weakly-bound supramolecular complexes. The (LS)DLPNO$\operatorname{CCSD}(T)$ and (NS)DLPNO-CCSD(T) schemes, based on the pair natural orbital (PNO) threshold settings, have been derived to provide accurate correlation energies for prototypical systems dominated by noncovalent interactions (i.e., the $\mathbf{5 6 6}$ dataset). The proposed schemes combine an accuracy comparable to DLPNO-CCSD(T) in its more stringent TightPNO setup with a less demanding computational cost. The good accuracyefficiency balance of the proposed strategies has allowed us to provide accurate interaction energies for the truxTTF $\bullet_{30} \mathrm{H}_{12}$ complex in its most stable arrangements, and to unveil the role of $\mathrm{CH}-\pi$ versus $\pi-\pi$ interactions at the high-level $a b$ initio DLPNO-CCSD(T).

\section{Theoretical Models and Computational Details}

Interaction energies, defined as the energy difference between the dimer and the constituting monomers at the minimum-energy geometry of the dimer, were calculated using the DLPNO-CCSD(T) approach. Note that the total energy of a molecular system can be divided into the Hartree-Fock energy $\left(E_{H F}\right)$ and the correlation energy $\left(E_{\text {corr }}\right)$. Therefore, the total interaction energy in a supramolecular complex $\left(E_{\text {int }}\right)$ may be defined as follows:

$$
E_{\mathrm{int}}=E_{H F, \text { int }}+E_{c o r r, \text { int }}
$$


where $E_{H F \text {,int }}$ and $E_{c o r r, \text { int }}$ are the Hartree-Fock and correlation components, respectively, to the interaction energy according to:

$$
\begin{aligned}
& E_{H F, \text { int }}=E_{H F}^{\text {dimer }}-\left(E_{H F}^{\text {monomer1 }}+E_{H F}^{\text {monomer } 2}\right) \\
& E_{c o r r, \text { int }}=E_{c o r r}^{\text {dimer }}-\left(E_{c o r r}^{\text {monomer1 }}+E_{c o r r}^{\text {monomer } 2}\right)
\end{aligned}
$$

In the DLPNO-CCSD(T) approach, ${ }^{[28]}$ three different levels of accuracy were initially proposed: cutoff settings defined in order of increasing accuracy as LoosePNO, NormalPNO, and TightPNO. The main parameters that change between the three accuracy levels are TCutPairs, TCutPNO, and TCutMKN, with their values given in Table 1. TCutPairs mainly defines which electron pairs are going to be treated at a lower (perturbation theory) level, TCutPNO states the size of the virtual space for each electron pair, and TCutMKN determines the size of the initial domain in which the PNOs are expanded. Whereas the Hartree-Fock energy is calculated by means of the standard selfconsistent field procedure and is not affected by the threshold parameters used in the DLPNO-CCSD(T) approach, these parameters directly impact on the calculated correlation energy. For a detailed description of the methodology, the reader is referred to the original literature. ${ }^{[27-28]}$

One possible drawback of the DLPNO ansatz is the underlying MP2 (Möller-Plesset perturbation theory up to second order) method used to estimate the energy contributions from weakly correlated electron pairs, an issue shared by most local correlation methods. This limits the accuracy of the method especially in treating supramolecular complexes, where many electron long-range pairs should be considered. The accuracy of the DLPNO approximation can be accordingly improved with a reasonable increase of computational cost by adjusting the cutoff threshold for the estimated pair correlation energies (TCutPairs) to $10^{-5} \mathrm{Eh}$. Note that the modification of the other cutoffs (TCutPNO and
TCutMKN) showed only a minor impact on the quantification of noncovalent interactions compared to the key TCutPairs threshold. ${ }^{[35]}$ The values for the settings used in our work with a tighter TCutPairs threshold (named as Loose2PNO and Normal2PNO) are also included in Table 1. ${ }^{[39]}$

Herein, we propose to estimate the DLPNO$\operatorname{CCSD}(\mathrm{T})$ correlation energy as a linear combination of the energies obtained with the standard and tighter TCutPairs cutoffs (Eq. 4 and 5),

$$
\begin{aligned}
& E_{\text {corr }}^{(L S D L P N O-C C S D(T)}=a \cdot E_{\text {corr }}^{D L P N O-C C S D(T)}(\text { LoosePNO })+ \\
& +(1-a) E_{\text {corr }}^{D L P O-C C S D(T)}(\text { Loose } 2 P N O)
\end{aligned}
$$

$$
\begin{aligned}
& E_{\text {corr }}^{(N S D L P N O-C C S D(T)}=b \cdot E_{\text {corr }}^{D L P N O-C C S D(T)}(\text { NormalPNO })+ \\
& (1-b) E_{\text {corr }}^{D L P O-C C S D(T)}(\text { Normal2PNO })
\end{aligned}
$$

where the contribution of each $E_{\text {corr }}^{D L P N O-C C S D(T)}$ term is weighted with a scaling factor $a$ (or $b$ ). Note that the LS and NS acronyms refer to the scaled protocols performed with the "Loose" and "Normal" setups, respectively.

Taking into account that basis set effects on the correlation energy $\left(E_{\text {corr }}\right)$ can be efficiently captured already with the MP2 method, we have extrapolated the energy calculated at the DLPNO-CCSD(T)/cc-pVTZ level to the complete basis set (CBS) limit by means of the following expression:

$$
\begin{aligned}
& E^{D L P N O-C C S D(T) / C B S}=E^{(H F / C B S)}+E_{c o r r}^{(M P 2 / C B S)}+ \\
& +\left(E_{c o r r}^{(X S) D L P N O-C C S D(T) / c c-p V T Z}-E_{c o r r}^{M P / c c-p V T Z}\right)= \\
& =E^{M P 2 / C B S}+\Delta E_{c o r r}^{D L P N O-C C S D(T)}
\end{aligned}
$$

as it has been done in previous works with much success. ${ }^{[29,40-43]}$ XS can adopt the LS or NS scaled protocols as explained above. Note that: 
Table 1. Values (in $E_{h}$ ) for the different thresholds of the DLPNO-CCSD(T) method employed in this work.

\begin{tabular}{|c|c|c|c|c|c|}
\hline & LoosePNO & NormalPNO & TightPNO & Loose2PNO & Normal2PNO \\
\hline TCutPairs & $10^{-3}$ & $10^{-4}$ & $10^{-5}$ & $10^{-5}$ & $10^{-5}$ \\
\hline TCutMKN & $10^{-3}$ & $10^{-3}$ & $10^{-4}$ & $10^{-3}$ & $10^{-3}$ \\
\hline TCutPNO & $10^{-6}$ & $3.33 \times 10^{-7}$ & $10^{-7}$ & $10^{-6}$ & $3.33 \times 10^{-7}$ \\
\hline
\end{tabular}

(i) HF and MP2 correlation energies are extrapolated to the complete basis set according to the Halkier et al. ${ }^{[44]}$ and Helgaker et al. ${ }^{[45]}$ two-point extrapolation schemes, respectively, using the Dunning's cc-pVTZ and cc-pVQZ basis sets, with $\alpha$ and $B$ exponents of 5.46 and 3.05 as reported in Ref. ${ }^{[46]}$; and (ii) the residual difference between DLPNO-CCSD(T) and MP2 correlation energies is assumed to have a less marked dependence on basis sets than the MP2 correlation energy itself.

The basis set superposition error (BSSE) was not counterpoise (CP) corrected for several reasons. First, the two-point CBS extrapolation scheme based on cc-pVTZ/cc-pVQZ basis sets approaches fairly well the CBS limit. Moreover, several studies have shown that the extrapolation scheme is either as or more accurate than the counterpoise correction for the evaluation of interaction energies and reaction barriers. ${ }^{[47-48]}$ We also refer the reader to recent studies on the accuracy of the extrapolation scheme applied for the evaluation of challenging experimental properties, ${ }^{[48-50]}$ or metal-ligand bond dissociations. ${ }^{[33,51]}$

Geometry optimizations of the corannulene/corannulene, TTF/corannulene, and truxTTF/hemifullerene dimers were performed at the DFT-D3 ${ }^{[52]}$ level with the hybrid revPBE0 ${ }^{[53-54]}$ exchange-correlation functional using the Becke-Johnson ${ }^{[55-57]}$ damping function -revPBEO-D3(BJ)- The correlation-consistent Dunning's cc-pVTZ basis set was used throughout. ${ }^{[58]}$

The computational effort was significantly reduced by making use of the resolution of the identity $(\mathrm{RI})^{[59]}$ with the corresponding matching auxiliary basis sets. ${ }^{[60]}$ For the DFT calculations, the chain-of-spheres $(\operatorname{COSX})^{[61]}$ technique was also employed for computing the exchange integrals. Large grids (Gridx6), especially required for the treatment of noncovalent interactions, were employed in the COSX procedure. The quadrature grids needed for numerical integration in DFT were also increased (Grid6) with respect to defaults, as strongly recommended for intermolecular interaction energies, as well as the corresponding thresholds for converging energies in the self-consistent field procedure (TightSCF). All the calculations were performed using the ORCA (version 3.0.0) program suite. ${ }^{[37]}$

\section{Calibration of the DLPNO-CCSD(T) settings}

In order to disentangle the effect of the TCutPairs threshold in the accurate treatment of noncovalent interactions, especially in dealing with $\pi-\pi$ and $\mathrm{CH}-\pi$ forces, the correlation contribution to the total interaction energy $\left(E_{\text {corr,int }}\right)$ in a benzene dimer was first evaluated. Figure 2 shows the $E_{\text {corr, int }}$ calculated for both parallel-displaced (PD) and the Tshaped (TS) dimers using different thresholds of DLPNO-CCSD $(T)$ upon increasing the intermolecular distance between benzene monomers (see Figure S1 for the total interaction energy profiles and Figure S2 for the interaction energy decomposition in the case of the PD dimer). The geometry of the minimumenergy structure of each benzene dimer was extracted from the 566 database, and the correlation energy of interaction was calculated along the intermolecular distance: from $d_{\mathrm{eq}}-1.0$ 
to $d_{\mathrm{eq}}+1.0 \AA$ in steps of $0.5 \AA$. $d_{\mathrm{eq}}$ is the intermolecular equilibrium distance between ring centroids calculated at 3.86 and $4.89 \AA$ for PD and TS benzene dimers, respectively.

The correlation energy of interaction calculated for the PD and TS benzene dimers at the reference DLPNO-CCSD(T)/cc-pVTZ (TightPNO) level is predicted to rapidly decay upon increasing the intermolecular distance (Figure

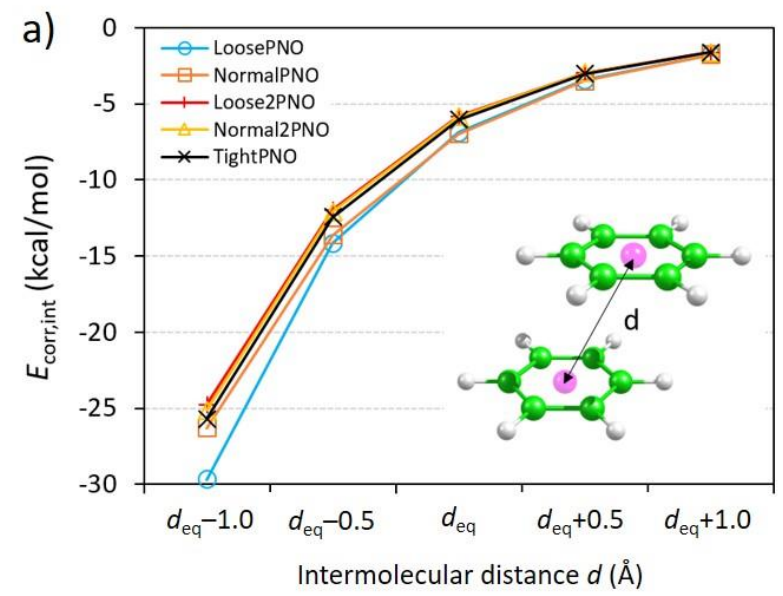

b)

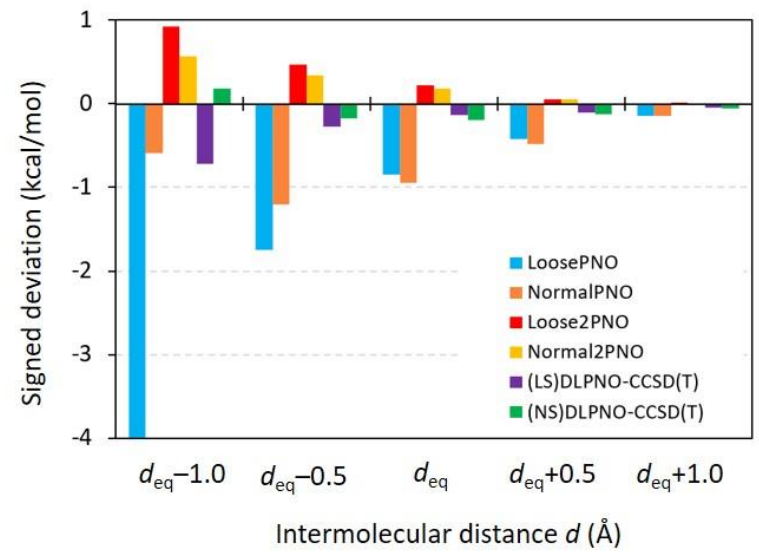

2a). $E_{\text {corr,int }}$ is calculated to be smaller, in absolute value, for the TS dimer due to the longer intermolecular distances resulting from $d_{\text {eq. }}$. By comparing the conventional three sets of default thresholds taking the TightPNO values as a reference, large deviations are predicted especially in the short range (Figure $2 b$ ). For example, in the PD dimer a large deviation of $-3.99 \mathrm{kcal} / \mathrm{mol}$ is predicted for LoosePNO at an intermolecular distance of $d_{\text {eq. }}-1.0=2.86 \AA$.
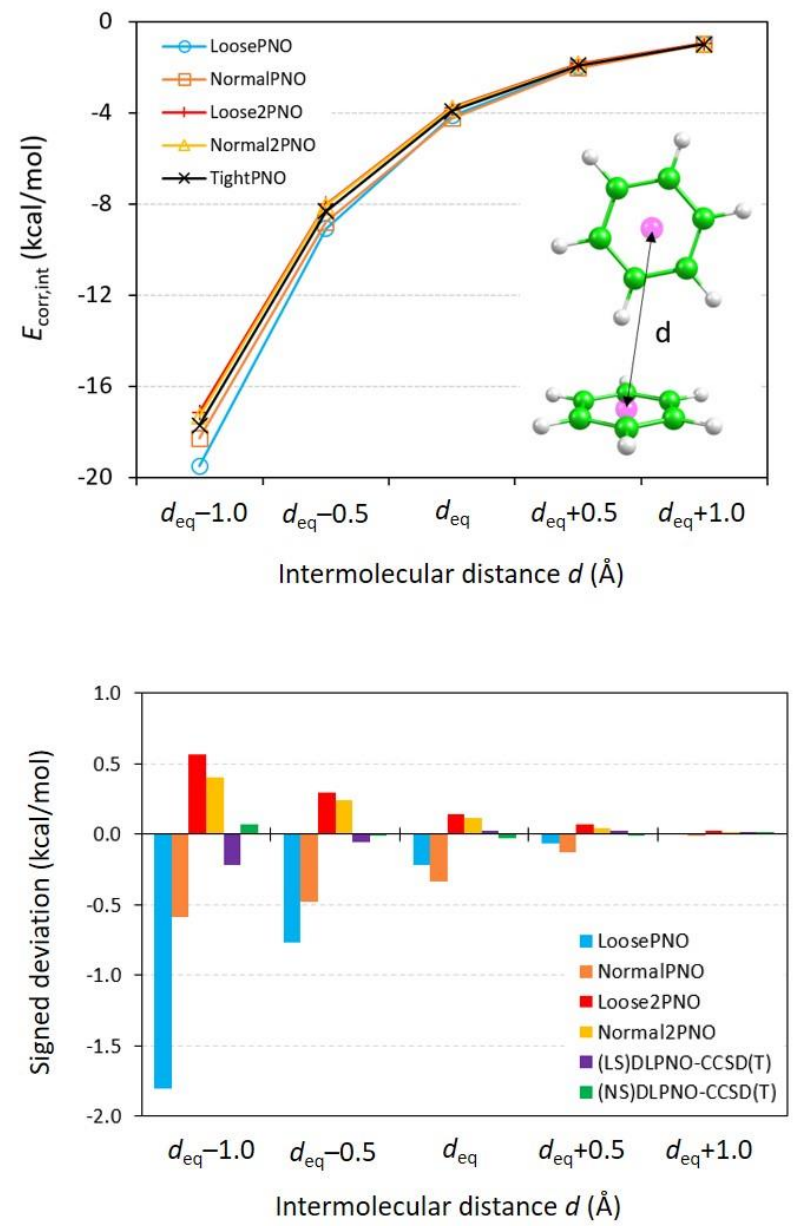

Figure 2. a) Correlation energy of interaction $\left(E_{\text {corr,int }}\right)$ as a function of the intermolecular distance calculated for parallel-displaced (left) and T-shaped (right) benzene dimers at the standard and modified PNO settings of DLPNO-CCSD(T)/cc-pVTZ. b) Signed $E_{\text {corr, int }}$ deviations with respect to the reference TightPNO setting, including the newly proposed LS and NS scaled settings.

This deviation rapidly diminishes upon separation of the benzene monomers and practically vanishes at $d_{\text {eq. }}+1.0=4.86 \AA(-0.14$ $\mathrm{kcal} / \mathrm{mol})$. For the TS dimer, the largest deviation computed from the reference energy is $-1.80 \mathrm{kcal} / \mathrm{mol}$ using the LoosePNO setup at 
an intermolecular distance of $d_{\text {eq }}-1.0=3.89 \AA$. In general, the NormalPNO setup provides lower deviations from TightPNO than LoosePNO, with a maximum error of 1.20 $\mathrm{kcal} / \mathrm{mol}$ calculated at $d=d_{\text {eq. }}-0.5=3.36 \AA$ in the PD dimer and of $0.59 \mathrm{kcal} / \mathrm{mol}$ at $d=$ $d_{\text {eq. }}-1.0=3.89 \AA$ in the TS dimer. Both LoosePNO and NormalPNO setups show a systematic overestimation of the correlation energy of interaction, which is computed globally larger at shorter intermolecular separations (Figure $2 \mathrm{~b}$ ).

$E_{\text {corr,int }}$ for the benzene dimers was also calculated at DLPNO-CCSD(T)/cc-pVTZ with the Loose2PNO and Normal2PNO setups (TCutPairs tightened to $10^{-5} E_{h}{ }^{[39]}$ see Table 1$)$. The asymptotic behavior of the $E_{c o r r, i n t}$ is correctly reproduced (Figure $2 \mathrm{a}$ ). In general, the modified setups perform better than the standard versions with the TCutPairs threshold $=10^{-3} E_{h}$ (LoosePNO) and $10^{-4} \mathrm{E}_{\mathrm{h}}$ (NormalPNO), with maximum deviations at $d_{\text {eq. }}-1.0 \AA$ of 0.93 and $0.56 \mathrm{kcal} / \mathrm{mol}$ for Loose2PNO and Normal2PNO, respectively, in the PD dimer, and of 0.57 and $0.40 \mathrm{kcal} / \mathrm{mol}$ in the TS dimer. The modified setups with TCutPairs of $10^{-5}$ show a systematic underestimation of $E_{\text {corr,int }}$ that is reduced upon increasing the intermolecular distance. A residual overestimation of only $-0.01 \mathrm{kcal} / \mathrm{mol}$ is predicted for Normal2PNO at $d_{\text {eq }}+1.0$ in the case of the PD dimer.

In view of these over/underestimation trends, we decided to linearly weight the $E_{\text {corr }}^{D L P N O-C C S D(T)}$ energy calculated with the default thresholds (LoosePNO and NormalPNO) and with the modified setups (Loose2PNO and Normal2PNO) in a controlled manner using Eq. 4 and 5 . The scaling factors $a$ and $b$ were preliminary set to $1 / 3$ for this initial exploration. Despite the empirical nature of the scaling factors, the scaled $E_{\text {corr,int }}^{(L S) D L P N O-C C S D(T)}$ and $E_{\text {corr,int }}^{(N S) D L P N O-C C S D(T)}$ energies show a consistent improvement in terms of the absolute deviations with respect to the reference at short-range intermolecular distances (Figure $2 b)$. For instance, largest deviations of 0.30 and $0.19 \mathrm{kcal} / \mathrm{mol}$ were calculated for (LS) and (NS)DLPNO-CCSD(T) setups, respectively, in the PD dimer. Likewise, negligible deviations lower than 0.20 and $0.07 \mathrm{kcal} / \mathrm{mol}$ were predicted, respectively, in the TS dimer.

\section{Optimization of the scaling factors for the S66 dataset}

In view of the improved performance upon scaling of the DLPNO-CCSD(T) correlation energies computed with the default and modified setups of the DLPNO approach, an optimization of the scaling factors $a$ and $b$ in Eq. 4 and 5 was performed by employing the S66 database. ${ }^{[62]}$ This database is especially designed for the treatment of noncovalent interactions and provides a balanced coverage of the different types of interactions usually found in organic molecules and biomolecules. It contains 66 noncovalently-bound complexes that can be divided into three subfamilies: hydrogen-bonded complexes, dispersiondominated systems, and 'mixed-character' structures. Reference correlation energies of interaction were calculated for the $\mathbf{S 6 6}$ dataset at the DLPNO-CCSD(T)/cc-pVTZ (TightPNO) level, which provides impressive mean, mean absolute and standard deviations of only -0.10 , 0.10 , and $0.06 \mathrm{kcal} / \mathrm{mol}$, respectively, compared with the 'gold-standard' $\operatorname{CCSD}(\mathrm{T})$ interaction energies. ${ }^{[35]}$ The $E_{\text {corr, int }}$ values calculated with the standard LoosePNO and NormalPNO setups, together with those computed with the modified analogues Loose2PNO and Normal2PNO, were used to obtain the rescaled versions (LS)DLPNO-CCSD(T) and (NS)DLPNO$\operatorname{CCSD}(T)$. The optimal scaling factors minimizing the mean absolute deviation (MAD) for the whole S66 dataset found for the "Loose" and "Normal" scaled setups are $a=0.48$ and $b=$ 
Table 2. Mean absolute deviation (MAD, in $\mathrm{kcal} / \mathrm{mol}$ ) of the DLPNO-CCSD $(\mathrm{T}) / \mathrm{cc}-\mathrm{pVTZ}$ correlation energies of interaction calculated for the S66 database at different PNO threshold settings, including the scaled settings (LS)DLPNO-CCSD(T) and (NS)DLPNO$\operatorname{CCSD}(\mathrm{T})$, with respect to the TightPNO reference.

\begin{tabular}{|c|c|c|c|c|c|c|}
\hline & Loose & Loose2 & $\begin{array}{c}(\mathrm{LS})^{a} \\
a=0.48\end{array}$ & Normal & Normal2 & $\begin{array}{c}(\mathrm{NS})^{a} \\
b=0.40\end{array}$ \\
\hline $\mathrm{H}$-bond & 0.380 & 0.236 & 0.305 & 0.174 & 0.158 & 0.164 \\
\hline Dispersion & 0.328 & 0.346 & 0.194 & 0.346 & 0.273 & 0.116 \\
\hline Mixed & 0.157 & 0.196 & 0.130 & 0.161 & 0.153 & 0.060 \\
\hline Total & 0.294 & 0.262 & 0.214 & 0.230 & 0.197 & 0.116 \\
\hline
\end{tabular}

0.40 , respectively. Small variations of the scaling parameters $a$ and $b$ from their optimized values do not dramatically impact on the accuracy, giving support to the stability of the scaled protocols (see Figure S5 and S6 in the Supporting Information). Total interaction energies calculated for the $\mathbf{S 6 6}$ database at the DLPNO-CCSD(T)/CBS level using the standard, modified, and scaled versions of the "Loose" and "Normal" PNO settings are gathered in Table S3, whereas signed deviations in $E_{\text {corr,int }}$ with respect to the "Tight" reference are summarized in Table S4 and Figure S3 and S4 (Supporting Information).

Table 2 summarizes the mean absolute deviation (MAD) values calculated for the $E_{\text {corr, int }}$ of the $\mathbf{S 6 6}$ database using the different versions of the PNO settings at the DLPNO-CCSD(T)/CCpVTZ level with respect to the TightPNO reference. In the $\mathrm{H}$-bonding family of complexes, Loose2PNO improves significantly the MAD obtained $(0.236 \mathrm{kcal} / \mathrm{mol})$ with respect to the standard LoosePNO $(0.380$ $\mathrm{kcal} / \mathrm{mol}$ ), whereas NormalPNO already performs really well, and Normal2PNO only reduces the MAD in $0.016 \mathrm{kcal} / \mathrm{mol}$. $E_{\text {corr,int }}$ for these electrostatically dominated systems is systematically underestimated by both "Loose" and "Normal" setups, either with standard or modified TCutPairs thresholds. The scaled PNO settings therefore provide MADs for $E_{\text {corr,int }}$ in between the standard and modified settings.

For the family of dispersion-dominated complexes, large deviations with MAD values of
0.328 and $0.346 \mathrm{kcal} / \mathrm{mol}$ are calculated for LoosePNO and NormalPNO, respectively (Table 2 ). This subfamily of compounds is especially important since the supramolecular assembly of most common D-A complexes based on fullerene and fullerene fragments are governed by these forces. The poor performance of LoosePNO and NormalPNO in this subset can be rationalized by considering that the nonbonding interactions rely on the MP2 corrections for the weak pairs, and MP2 is known to perform well for $\mathrm{H}$-bonded systems, yet it overestimates $\pi-\pi$ dispersion interactions. ${ }^{[26,}{ }^{63]}$ Whereas the increase of the TCutPairs to $10^{-5} E_{h}$ in Normal2PNO significantly improves the description of the dispersion-dominated systems with a MAD $=0.273 \mathrm{kcal} / \mathrm{mol}$, Loose2PNO (MAD $=0.346 \mathrm{kcal} / \mathrm{mol}$ ) worsens the results compared with standard LoosePNO. Noteworthy, standard settings provide a global overestimation of the interacting correlation energy, whereas modified setups with the more precise TCutPairs show an underestimation with respect to the reference values (Table S4, and Figure S3 and S4). This behavior agrees with the results discussed above for the PD and TS benzene dimers (see Figure 2). The new parametrized (LS)DLPNO-CCSD(T) and (NS)DLPNO-CCSD(T) versions therefore significantly improve the performance for the dispersion-dominated family of compounds with MADs of only 0.194 and $0.116 \mathrm{kcal} / \mathrm{mol}$, respectively. 
A similar behavior is predicted for the family governed by 'mixed' interactions. Modified settings worsen (in the case of Loose2PNO) or slightly improve (for Normal2PNO) the already small MADs of 0.157 and $0.161 \mathrm{kcal} / \mathrm{mol}$ obtained for LoosePNO and NormalPNO, respectively. Interestingly, the over/underestimation tendencies found for standard/modified settings (Table S4) lead to a significant reduction of MAD to 0.130 and 0.060 $\mathrm{kcal} / \mathrm{mol}$ for (LS)DLPNO-CCSD(T) and (NS)DLPNO-CCSD(T), respectively.

As shown in Table 2, a general improvement of the MAD calculated for the whole S66 database is obtained in going from standard LoosePNO $(0.294 \mathrm{kcal} / \mathrm{mol})$ and NormalPNO $(0.230 \mathrm{kcal} / \mathrm{mol})$ to the modified analogues Loose2PNO $(0.262 \mathrm{kcal} / \mathrm{mol})$ and Normal2PNO $(0.197 \mathrm{kcal} / \mathrm{mol})$. More importantly, the (LS)DLPNO-CCSD(T) and (NS)DLPNO-CCSD(T) schemes provide the best performance for the S66 set, with MADs of only 0.214 and 0.116 $\mathrm{kcal} / \mathrm{mol}$, respectively. Note that the accuracy of the scaled DLPNO-CCSD(T) protocols are roughly independent of the basis set since similar MADs are obtained for $\mathrm{S} 66$ using the ccpVTZ (Table 2) and aug-cc-pVTZ (Table S5) basis sets.

\section{Additional benchmarking: Medium-size supramolecular model dimers}

Model corannulene (cor $\bullet$ cor) homodimers and TTF•cor heterodimers (Figure 3 ) were designed to further validate the proposed scaled schemes (Eq. 4 and 5), and to assess the DLPNO$\operatorname{CCSD}(T) / c c-p V T Z$ performance with the standard and modified setups in large, yet affordable, supramolecular assemblies. Note that these supramolecular models contain noncovalent interactions similar to those governing the assembly of truxTTF with hemifullerene. The corannulene dimer was modeled in two different arrangements: bowlin-bowl (concave-convex) and staggered (concave-concave) dispositions (Figure $3 a$ and $3 b$, respectively), resembling those found in the trigonal and orthorhombic crystal polymorphs of hemifullerene. ${ }^{[64]}$ Similar dispositions are proposed for the TTF・cor heterodimer (Figure $3 c$ and $3 d$ ). These structures are analogous to the supramolecular structures computationally obtained for the truxTTF・ $\mathrm{C}_{30} \mathrm{H}_{12}$ dimer (vide infra, Figure 5). The four complexes were optimized at the revPBE0-D3(BJ)/cc-pVTZ level of theory, and the minimum-energy structures are shown in Figure 3 (see also Figure S7 in the Supporting Information). a)

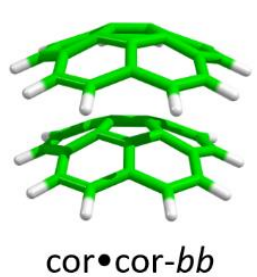

b)

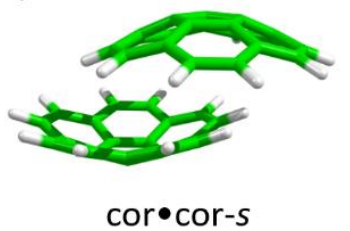

c)

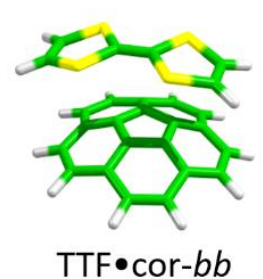

d)

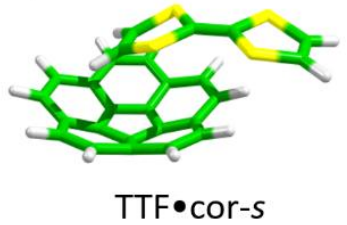

Figure 3. Supramolecular structures calculated at the revPBE0-D3(BJ)/cc-pVTZ level for the corannulene cor $\bullet$ cor homodimer ( $a$ and $b)$ and the TTF•cor heterodimer ( $c$ and $d$ ) in bowl-inbowl $(b b)$ and staggered $(s)$ arrangements.

An assessment of the DLPNO-CCSD(T) performance was undertaken by calculating the correlation energy of interaction ( $\left.E_{\text {corr,int }}\right)$ of the four supramolecular arrangements depicted in Figure 3 using the different PNO thresholds. Although the cor $\bullet$ cor homodimers and the $\pi \mathrm{TF} \bullet$ cor heterodimers exhibit a considerably large molecular size, reference interaction energies at DLPNO-CCSD(T)/cc-pVTZ (TightPNO) were also computed. Figure 4 shows the $E_{\text {corr,int }}$ values and the corresponding signed deviations with respect to the reference TightPNO energies (see Figure S8 in the Supporting Information for the total interaction energies).

$E_{\text {corr,int }}$ ranges from $-19.48 \mathrm{kcal} / \mathrm{mol}$ for TTF $\bullet$ cor- $\boldsymbol{b} \boldsymbol{b}$ to $-31.33 \mathrm{kcal} / \mathrm{mol}$ for cor $\bullet$ cor- $\boldsymbol{b} \boldsymbol{b}$ at the reference DLPNO-CCSD(T)/cc-pVTZ 
(TightPNO) level. Corannulene dimers are predicted with a larger $E_{\text {corr,int }}$ than the TTF・cor heterodimers due to the increased number of noncovalent interactions. Dimer cor $\bullet$ cor- $\boldsymbol{b} \boldsymbol{b}$ is calculated to provide the largest $E_{\text {corr,int, }}$ with a good concave-convex complementarity in which the $\pi-\pi$ interactions are maximized (short intermolecular contacts are calculated within 3.64-3.65 $\AA$, see Figure S7). Otherwise, structure cor $\bullet$ cor-s mostly interacts through $\mathrm{CH}-\pi$ forces with weaker $\pi-\pi$ contacts, providing an $E_{\text {corr,int }}$ as large as $-23.01 \mathrm{kcal} / \mathrm{mol}$. Interestingly, the concave-convex complementarity in TTF•cor-bb is not reflected in a larger $E_{\text {corr, int }}$ value with respect to TTF•cor-s (Figure $3 a$ ). In the latter, close $\mathrm{CH}-\pi$ contacts in the range of $2.67-3.11 \AA$ together with $\pi-\pi$ interactions (3.49-3.54 A, Figure 57$)$ result in an $E_{\text {corr,int }}$ of $-20.05 \mathrm{kcal} / \mathrm{mol}$ at the DLPNOCCSD(T)/cc-pVTZ (TightPNO) level.

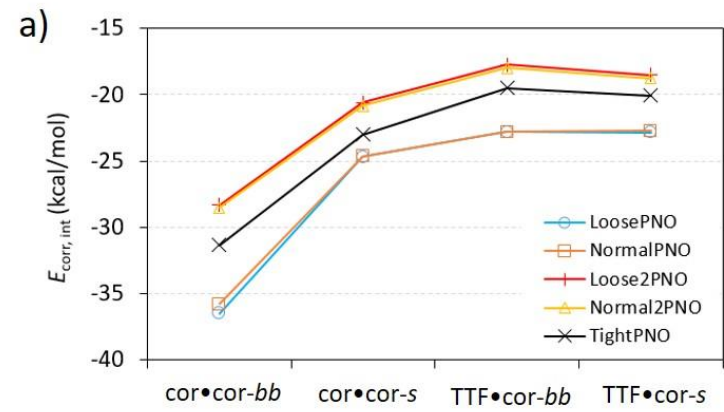

b)

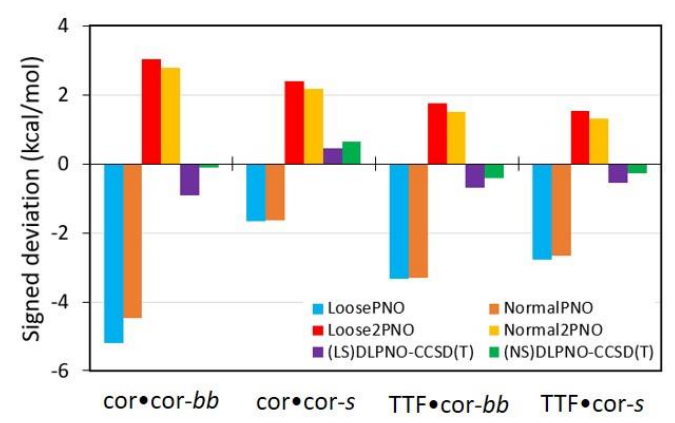

Figure 4. a) Correlation energy of interaction $\left(E_{\text {corr,int }}\right)$ calculated at the standard and modified PNO settings of DLPNO-CCSD(T)/cc-pVTZ for the cor•cor homodimers and the $T \mathrm{TF} \bullet \mathrm{cor}$ heterodimers. b) Signed $E_{c o r r, i n t}$ deviations with respect to the reference TightPNO setting, including the newly proposed LS and NS scaled settings.

Correlation energies of interaction calculated at DLPNO-CCSD(T)/cc-pVTZ with the standard LoosePNO and NormalPNO settings show a general overestimation of the interaction for the four supramolecular complexes (Figure 4a), with large deviations compared to the reference energies ranging from -1.66 and $-1.62 \mathrm{kcal} / \mathrm{mol}$ in cor-cor-s to -5.18 and -4.46 $\mathrm{kcal} / \mathrm{mol}$ in cor cor-bb, respectively (Figure $4 \mathrm{~b}$ ). As explained above, the weak interactions governing these complexes are captured in the standard settings by the MP2 corrections, which are well-known to overestimate dispersion forces. ${ }^{[26,}$ 63] However, by tightening the TCutPairs threshold to $10^{-5} \mathrm{E}_{\mathrm{h}}$ in the Loose2PNO and Normal2PNO setups, a consistent underestimation in the $E_{\text {corr, int }}$ is predicted in all systems with respect to the reference, with deviations ranging from 1.53 and $1.31 \mathrm{kcal} / \mathrm{mol}$ in TTF •cor-s to 3.03 and $2.79 \mathrm{kcal} / \mathrm{mol}$ in cor-cor-bb, respectively. The scaled schemes proposed herein outperform the previous settings with smaller deviations ranging from $0.45 \mathrm{kcal} / \mathrm{mol}$ in corecor-s to $-0.91 \mathrm{kcal} / \mathrm{mol}$ in cor-cor-bb for (LS)DLPNO-CCSD(T), and from $-0.11 \mathrm{kcal} / \mathrm{mol}$ in cor $\bullet$ cor-bb to $0.66 \mathrm{kcal} / \mathrm{mol}$ in cor-cor-s for (NS)DLPNO-CCSD(T). Importantly, the deviation calculated for the scaled settings seems to be independent of the size of the system or the nature of the supramolecular interaction, pointing toward accurate and sizeconsistent protocols.

Tables S6 and S7 in the Supporting Information summarize the partial and total timings and the specifications of each DLPNO$\operatorname{CCSD}(\mathrm{T})$ calculation as a function of the PNO settings for the two representative cor•cor-s and TTF•cor-s supramolecular complexes. Importantly, the scaled schemes (LS) and (NS)DLPNO-CCSD(T), which use normal and modified setups, are found faster in all cases with respect to TightPNO (see also Figure S9 and the respective discussion). Additionally, we expect that the speed-up of the scaled DLPNO- 
$\operatorname{CCSD}(\mathrm{T})$ versions with respect to DLPNOCCSD(T) (TightPNO) may be increased for large supramolecular complexes (see the Supporting Information for further discussion).

\section{Reference interaction energies for truxTTF• $\mathrm{C}_{30} \mathrm{H}_{12}$}
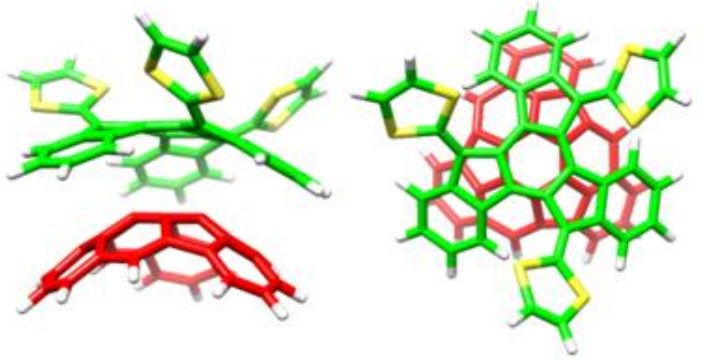

1
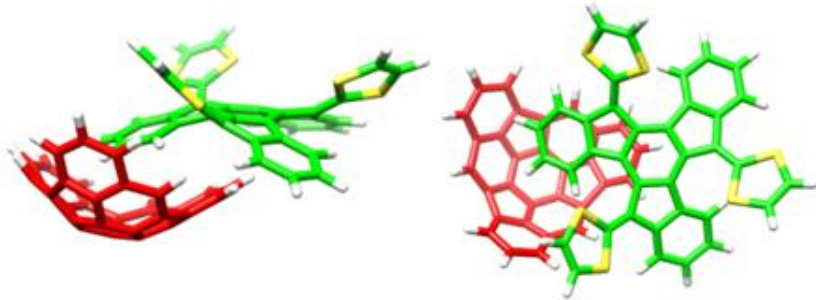

3
The truxTTF and $\mathrm{C}_{30} \mathrm{H}_{12}$ fragments may associate in at least four different dispositions to generate the supramolecular structures 1-4
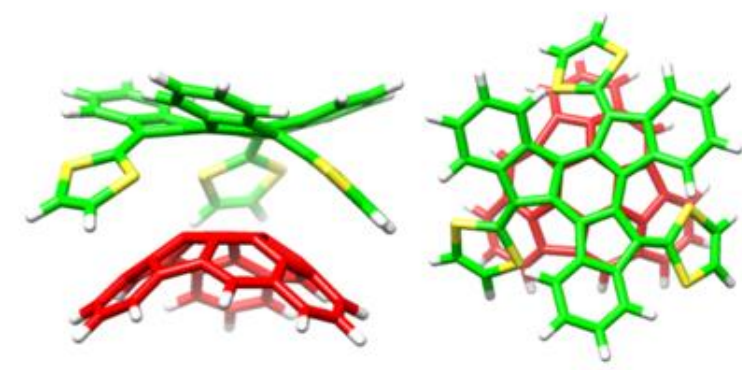

2

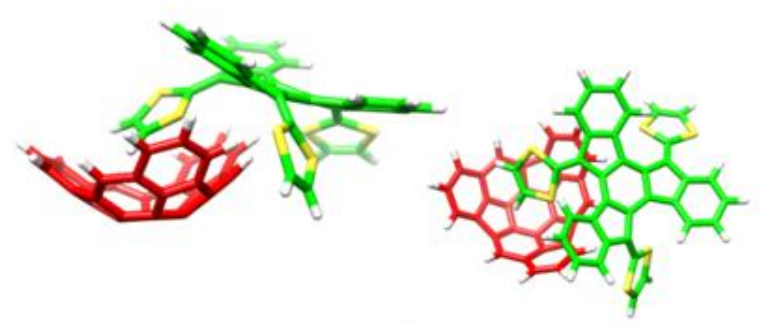

4

Figure 5. Minimum-energy structures computed for the truxTTF $\bullet \mathrm{C}_{30} \mathrm{H}_{12}$ heterodimer (1-4) at the revPBE0-D3(BJ)/cc-pVTZ level. Side (left) and top (right) views are displayed. truxTTF and $\mathrm{C}_{30} \mathrm{H}_{12}$ carbon atoms are colored in green and red, respectively.

depicted in Figure 5. These structures were rationally modeled resembling the arrangements found in the crystal packing of $\mathrm{C}_{30} \mathrm{H}_{12}$ in the two polymorphs reported by Scott et ${ }^{1}{ }^{[64]}$ and were calculated to be the most stable structures for the $\operatorname{truxTTF} \bullet \mathrm{C}_{30} \mathrm{H}_{12}$ associate at the dispersion-corrected DFT level. ${ }^{[10]}$ The initial geometries for the structures 1-4 were fully optimized at the revPBE0D3(BJ)/cc-pVTZ level.

In structures $\mathbf{1}$ and 2, the convex surface of $\mathrm{C}_{30} \mathrm{H}_{12}$ perfectly matches the two concave cavities of the truxTTF host, either through the cavity formed by the truxene core (1) or through the cavity formed by the three 1,3dithiole rings and the central benzene ring of the truxene core (2). Both structures are best described as bowl-in-bowl arrangements, where $\pi-\pi$ interactions are maximized, and resemble those found for the truxTTF・ $\mathrm{C}_{60}$ complex. ${ }^{[2,8]}$ Thus, the complexation driving force in $\mathbf{1}$ and $\mathbf{2}$ consists mainly of $\pi-\pi$ interactions with close C...C intermolecular distances in the 3.2-3.7 range (see Figure $\mathrm{S} 10$ in the Supporting Information). The intermolecular distance between the centroids of the central benzene rings is of 3.26 and $3.71 \AA$ for $\mathbf{1}$ and $\mathbf{2}$, respectively, suggesting a clear favorable interaction between the two moieties. These 
distances are significantly smaller than that calculated at the same level of theory for the bowl-in-bowl $\mathrm{C}_{30} \mathrm{H}_{12}$ homodimer $(4.07 \AA$ 趹, pointing to an adequate concave shape of truxTTF to host hemifullerene on both molecular sides. The short distances found between the centroid of the peripheral benzene rings of truxTTF in $\mathbf{1}$ or the dithiole rings in $\mathbf{2}$ and the closest carbon atom of $\mathrm{C}_{30} \mathrm{H}_{12}$ (3.28 and $3.37 \AA$, respectively) supports the complementary shape of both fragments.

A more complex scenario is found for the staggered host-guest structures, where different noncovalent interactions compete in the formation of the $\operatorname{truxTTF} \cdot \mathrm{C}_{30} \mathrm{H}_{12}$ associate. In structures $\mathbf{3}$ and $\mathbf{4}$, the concave cavities of truxTTF and $\mathrm{C}_{30} \mathrm{H}_{12}$ interact giving rise to heterodimers in which either a benzene (in $\mathbf{3}$ ) or a dithiole ring (in 4) of truxTTF is placed inside the concave cavity of the corannulene-based bowl (Figure 5). Although $\pi-\pi$ interactions between parallel rings would be expected to govern the host-guest complexation, the accurate revPBE0-D3(BJ)/cc-pVTZ optimized geometries show that $\mathrm{CH}-\pi$ interactions are essential in the stabilization of the associate. Short $\mathrm{H}$ (truxTTF) $\cdots \mathrm{C}\left(\mathrm{C}_{30} \mathrm{H}_{12}\right)$ and $\mathrm{H}\left(\mathrm{C}_{30} \mathrm{H}_{12}\right) \cdots \mathrm{C}$ (truxTTF) intermolecular contacts of around 2.50 and $2.70 \AA$, respectively, are computed for both $\mathbf{3}$ and $\mathbf{4}$, thus confirming the underlying role of the $\mathrm{CH}-\pi$ interactions in the staggered associates. In addition, some relatively short C...C and S...C intermolecular distances computed in the 3.50-3.70 $\AA$ range reveal the importance of other dispersion interactions in the formation of the complex (see Figure S10 for details).

The revPBE0-D3(BJ)/cc-pVTZ interaction energies $\left(E_{\text {int }}\right)$ recently reported for structures 1-4 show that the staggered heterodimers 3 and $\mathbf{4}$ are slightly more stable than their bowlin-bowl homologues 1 and 2 (Table 3). ${ }^{[10]}$ An energy difference of about $4 \mathrm{kcal} / \mathrm{mol}$ is computed between structures $\mathbf{1}$ and $\mathbf{3}$, where truxTTF approaches hemifullerene with the truxene face. The energy difference increases to a value of around $8 \mathrm{kcal} / \mathrm{mol}$ for structures 2 and 4, for which truxTTF approaches $\mathrm{C}_{30} \mathrm{H}_{12}$ with its dithiole rings. The stabilization of the staggered complexes relies on the number of $\mathrm{CH}-\pi$ interactions plus some contributing $\pi-\pi$ interactions. Surprisingly, among all structures modeled, the counterintuitive concave-concave heterodimer $\mathbf{4}$ was found to be the most stable structure with an interaction energy of -28.1 $\mathrm{kcal} / \mathrm{mol}$.

In order to confirm the energetic ordering predicted by DFT calculations for the different truxTTF $\bullet \mathrm{C}_{30} \mathrm{H}_{12}$ arrangements and to provide reference values, interaction energies for structures 1-4 were calculated using the accurate DLPNO-CCSD(T) approach with the scaled LS and NS protocols proposed in this work (Eq. 4 and 5). Note that DLPNOCCSD(T)/cc-pVTZ (TightPNO) calculations are computationally unfeasible due to the molecular size of the truxTTF $\bullet \mathrm{C}_{30} \mathrm{H}_{12}$ complex. The self-consistent field and correlation energies were extrapolated to the CBS limit using MP2 calculations at the cc-pVTZ and ccpVQZ basis sets according to a two-point extrapolation scheme (see Computational Details). The final DLPNO-CCSD(T)/CBS interaction energies were calculated according to Eq. 6 and are included in Table 3.

\begin{tabular}{|c|c|c|c|}
\hline Structure & revPBE0-D3(BJ)a & $\begin{array}{c}\text { (LS)DLPNO- } \\
\text { CCSD }(T)\end{array}$ & $\begin{array}{c}\text { (NS)DLPNO- } \\
\text { CCSD(T) }\end{array}$ \\
\hline 1 & -20.6 & -12.31 & -12.01 \\
\hline 2 & -20.1 & -17.56 & -16.42 \\
\hline 3 & -24.9 & -18.54 & -18.05 \\
\hline 4 & -28.1 & -21.03 & -20.51 \\
\hline
\end{tabular}

The interaction energies calculated using the scaled LS and NS protocols are consistent between them, and differ the most in only 1.14 $\mathrm{kcal} / \mathrm{mol}$ for structure $\mathbf{2}$ (Table 3 ). The concaveconvex arrangement 1 involving $\pi-\pi$ 
interactions between the carbon backbones is calculated to be the least stable structure $\left(E_{\text {int }} \sim\right.$ -12 kcal/mol), whereas structure 2 involving both $\mathrm{C} \cdots \mathrm{C}$ and $\mathrm{S} \cdots \mathrm{C} \pi-\pi$ interactions is computed around $5 \mathrm{kcal} / \mathrm{mol}$ more stable. This contrasts with the relative stabilities predicted at the revPBE0-D3(BJ)/cc-pVTZ level, which showed practically isoenergetic supramolecular interactions of $-20 \mathrm{kcal} / \mathrm{mol}$ for both structures. Moving to the staggered arrangements, $E_{\text {int }}$ values of -18.54 and $-18.05 \mathrm{kcal} / \mathrm{mol}$ are calculated for structure 3 at (LS)DLPNO-CCSD(T) and (NS)DLPNO-CCSD(T), respectively, whereas 4 is computed to be the most stable arrangement with $E_{\text {int }}$ of -21.03 and -20.51 $\mathrm{kcal} / \mathrm{mol}$, respectively. Again, the previously reported DFT-D3 calculations overestimate the interactions energies in $7-8 \mathrm{kcal} / \mathrm{mol}$. The systematic overbinding predicted by dispersion corrected DFT-D3 functionals has been recently reflected in the S12L training set, a database containing large supramolecular complexes dominated by noncovalent interactions. ${ }^{[43,65]}$

DLPNO-CCSD(T) calculations therefore confirm the concave-concave structure $\mathbf{4}$, in which a dithiole ring of truxTTF is placed in the basin of the $\mathrm{C}_{30} \mathrm{H}_{12}$ bowl and involves both $\mathrm{CH}-\pi$ and C...C and S...C $\pi-\pi$ interactions, as the most stable structure of the supramolecular truxTTF $\bullet \mathrm{C}_{30} \mathrm{H}_{12}$ complex. The presence of sulfur atoms in the truxTTF host therefore plays an important role in determining the relative stability of the supramolecular $\operatorname{truxTTF} \bullet \mathrm{C}_{30} \mathrm{H}_{12}$ structures: for both bowl-in-bowl and staggered arrangements, the structures presenting $\mathrm{S} \cdots \mathrm{C}$ $\pi-\pi$ interactions are more stable than those involving $\mathrm{C} \cdots \mathrm{C}$ interactions only. It should be noted that the computationally cheaper (LS)DLPNO-CCSD(T) scheme predicts interaction energies in good agreement with those obtained using the (NS)DLPNO-CCSD(T) scheme.

\section{Conclusions}

Herein, we present novel and simple linear scaled variants of the DLPNO-CCSD $(T)$ method to obtain accurate interaction energies in weakly-bound supramolecular complexes. An ostensible overestimation of most common dispersion forces, $\pi-\pi$ and $\mathrm{CH}-\pi$ interactions, in the $\mathrm{S} 66$ database has been detected with standard settings of DLPNO-CCSD(T) (LoosePNO and NormalPNO). Otherwise, an analogous underestimation of these interactions is predicted using the modified DLPNO-CCSD(T) variants where only the key parameter TCutPairs is augmented to $10^{-5} \mathrm{E}_{\mathrm{h}}$ (Loose2PNO and Normal2PNO). We have therefore introduced a scaling factor to weight the contribution of the standard and modified settings, leading to the schemes (LS)DLPNO$\operatorname{CCSD}(T)$ and (NS)DLPNO-CCSD(T). These schemes provide nearly TightPNO accuracy, which is essential for the quantification of weak noncovalent interactions, with a noticeable saving in computational cost. Importantly, the settings proposed in this work show a consistent accuracy irrespective of the nature and strength of the supramolecular interaction.

Accurate interaction energies calculated the (NS)DLPNO-CCSD(T)/CBS confirm the higher stability of the staggered concave-concave arrangements of the donor-acceptor truxTTF $\bullet \mathrm{C}_{30} \mathrm{H}_{12}$ heterodimer, highlighting the strength of $\mathrm{CH}-\pi$ forces in stabilizing supramolecular complexes. This is at odds with common concave-convex interactions between fullerene derivatives and their receptors, where only $\pi-\pi$ interactions are present. The hemifullerene and other carbon-based buckybowls are therefore expected to selfassemble with other donor compounds in a richer way than fullerenes do.

\section{Acknowledgments}

This work was supported by the Spanish Ministry of Economy and Competitiveness MINECO (CTQ2015-71154-P, CTQ2014-55073-P, CTQ2015-71936-REDT, and Unidad de Excelencia María de Maeztu MDM-2015-0538), the Generalitat Valenciana 
(PROMETEO/2016/135), and European FEDER funds (CTQ2015-71154-P). J.A. is grateful to MINECO for a "JdC-incorporación" postdoctoral fellowship. J.C. acknowledges the Ministerio de Educación, Cultura y Deporte (MECD) of Spain for a predoctoral FPU grant.

Keywords: dispersion forces; ab initio calculations; DLPNO-CCSD(T) scaled methods; donor-acceptor supramolecular complexes; noncovalent interactions

((Additional Supporting Information may be found in the online version of this article.))

\section{References and Notes}

[1] J. Cerny and P. Hobza, Phys. Chem. Chem. Phys. 2007, 9, 5291-5303.

[2] H. Isla, B. Grimm, E. M. Pérez, M. R. Torres, M. Á. Herranz, R. Viruela, J. Aragó, E. Ortí, D. M. Guldi and N. Martín, Chem. Sci. 2012, 3, 498-508.

[3] C. Romero-Nieto, R. García, M. Á. Herranz, L. Rodríguez-Pérez, M. SánchezNavarro, J. Rojo, N. Martín and D. M. Guldi, Angew. Chem. Int. Ed. 2013, 52, 10216-10220.

[4] C. Romero-Nieto, R. García, M. Á. Herranz, C. Ehli, M. Ruppert, A. Hirsch, D. M. Guldi and N. Martín, J. Am. Chem. Soc. 2012, 134, 9183-9192.

[5] S. S. Gayathri, M. Wielopolski, E. M. Pérez, G. Fernández, L. Sánchez, R. Viruela, E. Ortí, D. M. Guldi and N. Martín, Angew. Chem. Int. Ed. 2009, 48, 815-819.

[6] H. Isla, M. Gallego, E. M. Pérez, R. Viruela, E. Ortí and N. Martín, J. Am. Chem. Soc. 2010, 132, 1772-1773.

[7] B. Grimm, J. Santos, B. M. Illescas, A. Muñoz, D. M. Guldi and N. Martín, J. Am. Chem. Soc. 2010, 132, 17387-17389.

[8] E. M. Pérez, M. Sierra, L. Sánchez, M. R. Torres, R. Viruela, P. M. Viruela, E. Ortí and N. Martín, Angew. Chem. Int. Ed. 2007, 46, 18471851.

[9] A. de Juan, A. López-Moreno, J. Calbo, E. Ortí and E. M. Peréz, Chem. Sci. 2015, 6, 7008-7014.
[10] M. Gallego, J. Calbo, J. Aragó, R. M. Krick Calderon, F. H. Liquido, T. Iwamoto, A. K. Greene, E. A. Jackson, E. M. Pérez, E. Ortí, D. M. Guldi, L. T. Scott and N. Martín, Angew. Chem. Int. Ed. 2014, 53, 2170-2175.

[11] M. Gallego, J. Calbo, R. M. Krick Calderon, P. Pla, Y.-C. Hsieh, E. M. Pérez, Y.-T. Wu, E. Ortí, D. M. Guldi and N. Martín, Chem. Eur. J. DOI: 10.1002/chem.201604921.

[12] A. M. Sanders, T. J. Magnanelli, A. E. Bragg and J. D. Tovar, J. Am. Chem. Soc. 2016, 138, 3362-3370.

[13] T. Hasobe, Y. Kashiwagi, M. A. Absalom, J. Sly, K. Hosomizu, M. J. Crossley, H. Imahori, P. V. Kamat and S. Fukuzumi, Adv. Mater. 2004, 16, 975-979.

[14] L. Moreira, J. Calbo, B. M. Illescas, J. Aragó, I. Nierengarten, B. Delavaux-Nicot, E. Ortí, N. Martín and J.-F. Nierengarten, Angew. Chem. Int. Ed. 2015, 54, 1255-1260.

[15] L. Moreira, J. Calbo, R. M. Krick Calderon, J. Santos, B. M. Illescas, J. Aragó, J.-F. Nierengarten, D. M. Guldi, E. Ortí and N. Martín, Chem. Sci. 2015, 6, 4426-4432.

[16] L. Moreira, J. Calbo, J. Aragó, B. M. Illescas, I. Nierengarten, B. Delavaux-Nicot, E. Ortí, N. Martín and J.-F. Nierengarten, J. Am. Chem. Soc. 2016, DOI: 10.1021/jacs.1026b07250.

[17] M. A. Petrukhina, L. T. Scott and H. W. Kroto, Fragments of Fullerenes and Carbon Nanotubes: Designed Synthesis, Unusual Reactions, and Coordination Chemistry, Wiley, 2011, p. 440.

[18] L. T. Scott, Fragments of fullerenes: Novel syntheses, structures and reactions, 68, 1996, p. 291.

[19] H. Sakurai, T. Daiko and T. Hirao, Science 2003, 301, 1878.

[20] M. Nishio, M. Hirota and Y. Umezawa, The $\mathrm{CH} / \pi$ Interaction: Evidence, Nature, and Consequences, John Wiley \& Sons, 1998, p. 232.

[21] O. Takahashi, Y. Kohno and M. Nishio, Chem. Rev. 2010, 110, 6049-6076.

[22] M. Nishio, Phys. Chem. Chem. Phys. 2011, 13, 13873-13900.

[23] S. Grimme, Chem. Eur. J. 2012, 18, 9955-9964. 
[24] S. Grimme, WIREs Comput. Mol. Sci. 2011, 1, 211-228.

[25] K. E. Riley, M. Pitoňák, P. Jurečka and P. Hobza, Chem. Rev. 2010, 110, 5023-5063.

[26] P. Jurecka, J. Sponer, J. Cerny and P. Hobza, Phys. Chem. Chem. Phys. 2006, 8, 19851993.

[27] C. Riplinger and F. Neese, J. Chem. Phys. 2013, 138, 034106.

[28] C. Riplinger, B. Sandhoefer, A. Hansen and F. Neese, J. Chem. Phys. 2013, 139, 134101.

[29] D. G. Liakos, A. Hansen and F. Neese, J. Chem. Theory Comput. 2010, 7, 76-87.

[30] M. Sparta and F. Neese, Chem. Soc. Rev. 2014, 43, 5032-5041.

[31] J. Friedrich and J. Hänchen, J. Chem. Theory Comput. 2013, 9, 5381-5394.

[32] A. Hansen, C. Bannwarth, S. Grimme, P. Petrović, C. Werlé and J.-P. Djukic, ChemistryOpen 2014, 3, 177-189.

[33] M. Sparta, C. Riplinger and F. Neese, J. Chem. Theory Comput. 2014, 10, 1099-1108.

[34] C. Riplinger, M. D. Sampson, A. M. Ritzmann, C. P. Kubiak and E. A. Carter, J. Am. Chem. Soc. 2014, 136, 16285-16298.

[35] D. G. Liakos, M. Sparta, M. K. Kesharwani, J. M. L. Martin and F. Neese, J. Chem. Theory Comput. 2015, 11, 1525-1539.

[36] D. G. Liakos and F. Neese, J. Chem. Theory Comput. 2015, 11, 4054-4063.

[37] F. Neese, WIREs Comput. Mol. Sci. 2012, 2, 73-78.

[38] D. Maganas, M. Roemelt, M. Havecker, A. Trunschke, A. Knop-Gericke, R. Schlogl and F. Neese, Phys. Chem. Chem. Phys. 2013, 15, $7260-$ 7276.

[39] H. Kruse, A. Mladek, K. Gkionis, A. Hansen, S. Grimme and J. Sponer, J. Chem. Theory Comput. 2015, 11, 4972-4991.

[40] M. O. Sinnokrot, E. F. Valeev and C. D. Sherrill, J. Am. Chem. Soc. 2002, 124, 1088710893.

[41] P. Jurečka and P. Hobza, Chem. Phys. Lett. 2002, 365, 89-94.

[42] D. G. Liakos and F. Neese, J. Phys. Chem. A 2012, 116, 4801-4816.
[43] J. Calbo, E. Ortí, J. C. Sancho-García and J. Aragó, J. Chem. Theory Comput. 2015, 11, 932-939.

[44] A. Halkier, T. Helgaker, P. Jørgensen, W. Klopper and J. Olsen, Chem. Phys. Lett. 1999, 302, 437-446.

[45] T. Helgaker, W. Klopper, H. Koch and J. Noga, J. Chem. Phys. 1997, 106, 9639-9646.

[46] F. Neese and E. F. Valeev, J. Chem. Theory Comput. 2011, 7, 33-43.

[47] L. A. Burns, M. S. Marshall and C. D. Sherrill, J. Chem. Theory Comput. 2014, 10, 4957.

[48] J. R. Alvarez-Idaboy and A. Galano, Theor. Chem. Acc. 2010, 126, 75-85.

[49] A. Vega-Rodriguez and J. R. AlvarezIdaboy, Phys. Chem. Chem. Phys. 2009, 11, 7649-7658.

[50] M. Gruber-Stadler, M. Mühlhäuser, S. R. Sellevåg and C. J. Nielsen, J. Phys. Chem. A 2008, 112, 9-22.

[51] X. Xu, W. Zhang, M. Tang and D. G. Truhlar, J. Chem. Theory Comput. 2015, 11, 2036-2052.

[52] S. Grimme, J. Antony, S. Ehrlich and H. Krieg, J. Chem. Phys. 2010, 132, 154104.

[53] C. Adamo and V. Barone, J. Chem. Phys. 1999, 110, 6158-6170.

[54] Y. Zhang and W. Yang, Phys. Rev. Lett. 1998, 80, 890-890.

[55] E. R. Johnson and A. D. Becke, J. Chem. Phys. 2005, 123, 024101-024107.

[56] A. D. Becke and E. R. Johnson, J. Chem. Phys. 2005, 122, 154104.

[57] S. Grimme, S. Ehrlich and L. Goerigk, J. Comput. Chem. 2011, 32, 1456-1465.

[58] J. T. H. Dunning, J. Chem. Phys. 1989, 90, 1007-1023.

[59] K. Eichkorn, O. Treutler, H. Öhm, M. Häser and R. Ahlrichs, Chem. Phys. Lett. 1995, 240, 283-290.

[60] K. Eichkorn, F. Weigend, O. Treutler and R. Ahlrichs, Theor. Chem. Acc. 1997, 97, 119124.

[61] F. Neese, F. Wennmohs, A. Hansen and U. Becker, Chem. Phys. 2009, 356, 98-109.

[62] J. Řezáč, K. E. Riley and P. Hobza, J. Chem. Theory Comput. 2011, 7, 2427-2438. 
[63] S. M. Cybulski and M. L. Lytle, J. Chem. Phys. 2007, 127, 141102.

[64] M. A. Petrukhina, K. W. Andreini, L. Peng and L. T. Scott, Angew. Chem. Int. Ed. 2004, 116, 5593-5597.
[65] J. Calbo, E. Ortí, J. C. Sancho-García and J. Aragó, Chapter Two - The Nonlocal Correlation Density Functional VV10: A Successful Attempt to Accurately Capture Noncovalent Interactions, 11 (Ed. A. D. David), Elsevier, 2015, pp. 37-102. 


\section{GRAPHICAL ABSTRACT}

AUTHOR NAMES

Joaquín Calbo, ${ }^{[a], *}$ Juan C. Sancho-García, ${ }^{[b]}$ Enrique Orti, ${ }^{[a]}$ Juan Aragó ${ }^{[a], *}$

TITLE

DLPNO-CCSD(T) Scaled Methods for the Accurate Treatment of Large Supramolecular Complexes

TEXT

In this work, scaled variants of the DLPNO-CCSD $(T)$ approach are presented as accurate yet affordable $a b$ initio protocols to deal with noncovalent interactions. The scaled DLPNO-CCSD(T) methodologies provide nearly TightPNO accuracy at a reduced computational cost. These new protocols are used to disentangle the energetic ordering of the possible arrangements in a large donor-acceptor supramolecular assembly. Calculations indicate that buckybowls are able to interact with electron-donor entities in a richer way than fullerenes do.

\section{GRAPHICAL ABSTRACT FIGURE}

\section{Scaled DLPNO-CCSD(T)}

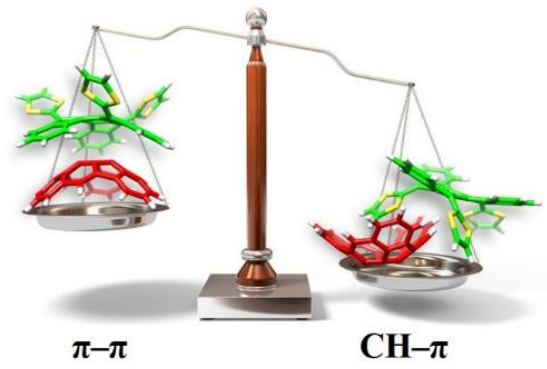

